\title{
Primordial magnetic fields and nonlinear electrodynamics
}

\author{
Kerstin E. Kunze1 1 \\ Departamento de Física Fundamental, \\ and \\ Instituto Universitario de Física Fundamental y Matemáticas (IUFFyM), \\ Universidad de Salamanca, \\ Plaza de la Merced s/n, E-37008 Salamanca, Spain
}

\begin{abstract}
The creation of large scale magnetic fields is studied in an inflationary universe where electrodynamics is assumed to be nonlinear. After inflation ends electrodynamics becomes linear and thus the description of reheating and the subsequent radiation dominated stage are unaltered. The nonlinear regime of electrodynamics is described by lagrangians having a power law dependence on one of the invariants of the electromagnetic field. It is found that there is a range of parameters for which primordial magnetic fields of cosmologically interesting strengths can be created.
\end{abstract}

\section{Introduction}

Magnetic fields are observed to be associated with most structures in the universe. Observations indicate magnetic fields on stellar upto supergalactic scales. The field strengths vary from a few $\mu \mathrm{G}$ on galactic scale, upto $10^{3} \mathrm{G}$ for solar type stars and upto $10^{13} \mathrm{G}$ for neutron stars. Furthermore, the magnetic field structure depends on the object it is associated with. Thus, e.g., magnetic fields observed in elliptical galaxies show a different structure from those associated with spiral galaxies [1].

Magnetic fields in stars can be explained by the formation of protostars out of condensed interstellar matter which was pervaded by a pre-existing large scale magnetic field (see, e.g., [2]). An open problem remains to explain the origin of such large scale magnetic fields.

There are different types of proposals. Ranging from processes on small scales, such as vortical perturbations and phase transitions to models taking advantage of the possibility of amplifying perturbations in the electromagnetic field during inflation in the early universe (see, e.g., [3]).

Inflation provides a mechanism to amplify perturbations in some field to appreciable size. In order for this mechanism to lead to primordial magnetic seed fields of cosmologically interesting strength, the corresponding lagrangian should not be conformally invariant. The Maxwell lagrangian describing linear electrodynamics is conformally invariant. There have been already a multitude of proposals to break the conformal invariance of the Maxwell theory [4], e.g. by coupling

\footnotetext{
${ }^{1}$ E-mail: kkunze@usal.es, Kerstin.Kunze@cern.ch
} 
to a scalar field [5], breaking Lorentz invariance [6], adding extra dimensions [7] or a coupling to curvature terms [8].

Here nonlinear electrodynamics is considered. It has its origins in the search for a classical singularity-free theory of the electron by Born and Infeld [9]. Later on it was realized that virtual electron pair creation induces a self-coupling of the electromagnetic field. For slowly varying, but arbitrarily strong electromagnetic fields the self-interaction energy was computed by Heisenberg and Euler (cf. [10]-[12]).

The propagation of a photon in an external electromagnetic field can be described effectively by the Heisenberg-Euler langrangian. Moreover, the transition amplitude for photon splitting in quantum electrodynamics is nonvanishing in this case. In principle, this might lead to observational effects, e.g., on the electromagnetic radiation coming from neutron stars which are known to have strong magnetic fields. [12, 13]. In particular, certain features in the spectra of pulsars can be explained by photon splitting [14].

Finally, Born-Infeld type actions also appear as a low energy effective action of open strings [15, 16]. As was shown in [17] the low energy dynamics of D-branes is described by the Dirac-BornInfeld action.

The model of the cosmological background that will be considered consists of a stage of de Sitter inflation followed by reheating and a standard radiation dominated stage. Quantum fluctuations in the electromagnetic field are excited within the horizon during inflation. Once outside the horizon they become classical perturbations. As mentioned above, in general, the conformal invariance of the four dimensional Maxwell field has to be broken in order to amplify the perturbations in the electromagnetic field significantly. Thus, here electrodynamics is considered to be nonlinear during the de Sitter stage. This could be motivated by the presence of possible quantum corrections to quantum electrodynamics at high energies. However, once inflation ends electrodynamics is described by standard Maxwell electrodynamics. Thus the subsequent evolution described by the standard model of cosmology is unchanged.

\section{Nonlinear electrodynamics in the early universe}

The Born-Infeld or Heisenberg-Euler lagrangians provide particular examples of theories of nonlinear electrodynamics. In general the action of nonlinear electrodynamics coupled minimally to gravity can be written as, see e.g. [16, 18]

$$
S=\frac{1}{16 \pi G_{N}} \int d^{4} x \sqrt{-g} R+\frac{1}{4 \pi} \int d^{4} x \sqrt{-g} L(X, Y),
$$

where $L(X, Y)$ is the lagrangian of nonlinear electrodynamics. Furthermore, the invariants are denoted by $X \equiv \frac{1}{4} F_{\mu \nu} F^{\mu \nu}$ and $Y \equiv \frac{1}{4} F_{\mu \nu}{ }^{*} F^{\mu \nu}$, where ${ }^{*} F^{\mu \nu}$ is the dual bi-vector given by ${ }^{*} F^{\mu \nu}=\frac{1}{2 \sqrt{-g}} \epsilon^{\mu \nu \alpha \beta} F_{\alpha \beta}$, and $\epsilon^{\mu \nu \alpha \beta}$ the Levi-Civita tensor with $\epsilon_{0123}=+1$.

The equations of motion are given by

$$
\nabla_{\mu} P^{\mu \nu}=0
$$

where $P_{\mu \nu}=-\left(L_{X} F_{\mu \nu}+L_{Y}{ }^{*} F_{\mu \nu}\right)$, furthermore $L_{A}$ denotes $L_{A}=\partial L / \partial A$, and

$$
\nabla_{\mu}^{*} F^{\mu \nu}=0
$$


which implies that $F_{\mu \nu}=\partial_{\mu} A_{\nu}-\partial_{\nu} A_{\mu}$. The notation used is given by $\mu, \nu . .=0 \ldots 3$ and $i, j . .=$ $1,2,3$. Moreover, the electromagnetic field is treated as a perturbation so that the vacuum Einstein equations apply to the background cosmology. The background metric is chosen to be of the form

$$
d s^{2}=a^{2}(\eta)\left[-d \eta^{2}+d \mathbf{x}^{2}\right]
$$

Furthermore, following [4] the Maxwell tensor is written in terms of the electric and magnetic fields, $\vec{E}$ and $\vec{B}$, respectively, as follows,

$$
F_{\mu \nu}=a^{2}\left(\begin{array}{cccc}
0 & -E_{x} & -E_{y} & -E_{z} \\
E_{x} & 0 & B_{z} & -B_{y} \\
E_{y} & -B_{z} & 0 & B_{x} \\
E_{z} & B_{y} & -B_{x} & 0
\end{array}\right) .
$$

These are the components in the "lab" frame in which in linear electrodynamics the frozen-in magnetic field decays in an expanding universe as $1 / a^{2}$.

Then equations (2.2) and (2.3) imply

$$
\begin{aligned}
\nabla \cdot \vec{E}+\frac{\left(\nabla L_{X}\right) \cdot \vec{E}}{L_{X}}-\frac{\left(\nabla L_{Y}\right) \cdot \vec{B}}{L_{X}} & =0 \\
\frac{1}{a^{2}} \partial_{\eta}\left(a^{2} \vec{E}\right)-\nabla \times \vec{B}+\frac{\partial_{\eta} L_{X}}{L_{X}} \vec{E}-\frac{\partial_{\eta} L_{Y}}{L_{X}} \vec{B}-\frac{\left(\nabla L_{X}\right) \times \vec{B}}{L_{X}}-\frac{\left(\nabla L_{Y}\right) \times \vec{E}}{L_{X}} & =0 \\
\nabla \cdot \vec{B} & =0 \\
\frac{1}{a^{2}} \partial_{\eta}\left(a^{2} \vec{B}\right)+\nabla \times \vec{E} & =0
\end{aligned}
$$

Although Maxwell's equations are recovered for lagrangians of the form $L=n_{0} X+n_{1} Y$, where $n_{0}$ and $n_{1}$ are constants, standard linear electrodynamics corresponds to $L=-X$. Equations (2.6) to (2.9) is a set of four first order partial differential equations which can be transformed into a set of two second order partial differential equations. This describes the evolution of the nonlinear electromagnetic field in a curved background. In linear electrodynamics this procedure leads to two decoupled wave equations, one for the magnetic and one for the electric field. In the case of nonlinear electrodynamics the resulting wave equations for the electric and the magnetic fields are no longer decoupled because of the nonlinearities.

Taking the curl of equation (2.7) and using equations (2.8) and (2.9) a wave type equation for the magnetic field $\vec{B}$ can be found.

$$
\begin{aligned}
& \frac{1}{a^{2}} \frac{\partial^{2}}{\partial \eta^{2}}\left(a^{2} \vec{B}\right)+\frac{1}{a^{2}} \frac{\partial_{\eta} L_{X}}{L_{X}} \partial_{\eta}\left(a^{2} \vec{B}\right)+\frac{1}{a^{2}} \frac{\partial_{\eta} L_{Y}}{L_{X}} \partial_{\eta}\left(a^{2} \vec{E}\right)+\frac{\partial_{\eta} L_{Y}}{L_{X}}\left(\frac{\partial_{\eta} L_{X}}{L_{X}} \vec{E}-\frac{\partial_{\eta} L_{Y}}{L_{X}} \vec{B}\right) \\
- & \Delta \vec{B}+\vec{E} \times \nabla\left(\frac{\partial_{\eta} L_{X}}{L_{X}}\right)-\vec{B} \times \nabla\left(\frac{\partial_{\eta} L_{Y}}{L_{X}}\right)-\frac{\partial_{\eta} L_{Y}}{L_{X}}\left[\frac{\left(\nabla L_{X}\right) \times \vec{B}}{L_{X}}+\frac{\left(\nabla L_{Y}\right) \times \vec{E}}{L_{X}}\right] \\
+ & \nabla \times\left[\frac{\left(\nabla L_{X}\right) \times \vec{B}}{L_{X}}\right]+\nabla \times\left[\frac{\left(\nabla L_{Y}\right) \times \vec{E}}{L_{X}}\right]=0
\end{aligned}
$$


Similarly, taking the time derivative of of equation (2.7) and using the remaining equations results in a wave type equation for the electric field $\vec{E}$,

$$
\begin{aligned}
& \frac{\partial^{2}}{\partial \eta^{2}}\left(a^{2} \vec{E}\right)+\partial_{\eta}\left[\frac{\partial_{\eta} L_{X}}{L_{X}} a^{2} \vec{E}\right]-\partial_{\eta}\left[\frac{\partial_{\eta} L_{Y}}{L_{X}} a^{2} \vec{B}\right] \\
- & \Delta\left(a^{2} \vec{E}\right)-\partial_{\eta}\left[\frac{\left(\nabla L_{X}\right) \times a^{2} \vec{B}}{L_{X}}\right]-\partial_{\eta}\left[\frac{\left(\nabla L_{Y}\right) \times a^{2} \vec{E}}{L_{X}}\right] \\
- & \nabla\left[\frac{\left(\nabla L_{X}\right) \cdot\left(a^{2} \vec{E}\right)}{L_{X}}\right]+\nabla\left[\frac{\left(\nabla L_{Y}\right) \cdot\left(a^{2} \vec{B}\right)}{L_{X}}\right]=0
\end{aligned}
$$

Equations (2.10) and (2.11), respectively, are coupled and nonlinear which makes it quite difficult to find exact solutions. However, as a first approximation it might be interesting to find the behaviour of the magnetic field neglecting the spatial dependence. This is the long wavelength approximation. Considering variations over a characteristic comoving length scale $L$ much larger than the horizon $a H$ then the spatial derivatives of a quantity can be neglected with respect to its time derivatives (see for example, [19]). In general, $\vec{E}$ and $\vec{B}$ can be written in terms of Fourier expansions,

$$
\vec{E}(\vec{x}, \eta)=\int d^{3} k e^{i \vec{k} \cdot \vec{x}} \vec{E}_{k}(\eta) \quad \vec{B}(\vec{x}, \eta)=\int d^{3} k e^{i \vec{k} \cdot \vec{x}} \vec{B}_{k}(\eta) .
$$

Thus in the long wavelength approximation effectively only modes with small wave numbers will contribute to the Fourier expansions. Therefore, e.g. $\vec{B}(\vec{x}, \eta) \simeq \int_{0}^{k_{c}} d^{3} k e^{i \vec{k} \cdot \vec{x}} \vec{B}_{k}(\eta)$. Just using one mode $e^{i \vec{k} \cdot \vec{x}} \vec{B}_{k}(\eta)$ for $k \ll k_{c} \lesssim a H$ one can show that in the limit $k \rightarrow 0$ the terms involving spatial derivatives become subleading. As it is commonly done, this approximation is applied to the second order equations (2.10) and (2.11) (cf., for example, [4]).

A different way of looking at this is to use the stochastic approach to inflation where the mode expansion of a field, for example the inflaton, is separated into modes larger than the coarse graining domain and modes with wavelengths smaller than the coarse graining scale [20]. The superhorizon modes contribute to the coarse grained field which is made homogeneous by averaging over the coarse graining domain. The effect of modes leaving the coarse graining domain and contributing to the coarse grained field can effectively be modeled by a noise term in the equation of the coarse grained field. Neglecting this backreaction effect the dynamics of the field on superhorizon scales is basically described by the homogeneous, coarse grained field.

Thus neglecting spatial derivatives equation (2.10) implies,

$$
\overrightarrow{\mathcal{B}}_{k}^{\prime \prime}+\frac{L_{X}^{\prime}}{L_{X}} \overrightarrow{\mathcal{B}}_{k}^{\prime}+\frac{L_{Y}^{\prime}}{L_{X}} \overrightarrow{\mathcal{E}}_{k}^{\prime}+\frac{L_{Y}^{\prime}}{L_{X}}\left[\frac{L_{X}^{\prime}}{L_{X}} \overrightarrow{\mathcal{E}}_{k}-\frac{L_{Y}^{\prime}}{L_{X}} \overrightarrow{\mathcal{B}}_{k}\right]=0
$$

where $\overrightarrow{\mathcal{B}}_{k} \equiv a^{2} \vec{B}_{k}, \overrightarrow{\mathcal{E}}_{k} \equiv a^{2} \vec{E}_{k}$ and a prime denotes the derivative with respect to conformal time $\eta$, that is ${ }^{\prime} \equiv \frac{d}{d \eta}$. In the case where the lagrangian only depends on $X, L_{Y}=0, \overrightarrow{\mathcal{B}}_{k}=$ const. is a solution which corresponds to the conformally invariant case, that is linear electrodynamics. In general, for $L_{Y}=0$, equation (2.13) implies

$$
\overrightarrow{\mathcal{B}_{k}^{\prime}}=\frac{\vec{K}_{k}}{L_{X}},
$$


where $\vec{K}_{k}$ is a constant vector and $L_{X} \neq 0$. Moreover, linear electrodynamics is recovered for $\vec{K}_{k} \equiv 0$.

Furthermore, equation (2.11) implies

$$
\frac{d}{d \eta}\left[\overrightarrow{\mathcal{E}}_{k}^{\prime}+\frac{L_{X}^{\prime}}{L_{X}} \overrightarrow{\mathcal{E}}_{k}-\frac{L_{Y}^{\prime}}{L_{X}} \overrightarrow{\mathcal{B}}_{k}\right] \simeq 0
$$

Equation (2.15) can be integrated to give

$$
\overrightarrow{\mathcal{E}}_{k}^{\prime}+\frac{L_{X}^{\prime}}{L_{X}} \overrightarrow{\mathcal{E}}_{k}-\frac{L_{Y}^{\prime}}{L_{X}} \overrightarrow{\mathcal{B}}_{k}=\vec{P}_{k}
$$

where $\vec{P}_{k}$ is a constant vector. The homogeneous part of equations (2.10) and (2.11) are coupled non trivially because of $L_{Y}$, cf. equations (2.13) and (2.15) . Therefore in order to find solutions, the Lagrangian will be considered to be only a function of $X, L=L(X)$. Furthermore, since $X=\frac{1}{2}\left(\vec{B}^{2}-\vec{E}^{2}\right)$ it is useful to find equations for $\overrightarrow{\mathcal{E}}_{k}^{2}$ and $\overrightarrow{\mathcal{B}}_{k}^{2}$ which are given by, for $\vec{P}_{k}^{2}>0$,

$$
\begin{aligned}
& \overrightarrow{\mathcal{E}}_{k}^{2 \prime \prime}+3 \frac{L_{X}^{\prime}}{L_{X}} \overrightarrow{\mathcal{E}}_{k}^{2 \prime}+2 \frac{L_{X}^{\prime \prime}}{L_{X}} \overrightarrow{\mathcal{E}}_{k}^{2}=2 \vec{P}_{k}^{2} \\
& \overrightarrow{\mathcal{B}}_{k}^{2 \prime \prime}+\frac{L_{X}^{\prime}}{L_{X}} \overrightarrow{\mathcal{B}}_{k}^{2 \prime}-2 \frac{\vec{K}_{k}^{2}}{L_{X}^{2}}=0 .
\end{aligned}
$$

Assuming that the constant vector in equation (2.16) vanishes, $\vec{P}_{k}=0$, leads to a significant simplification. In this case, equation (2.16) for $L=L(X)$ can be solved immediately, giving for the electric field

$$
\overrightarrow{\mathcal{E}}_{k}=\frac{\vec{M}_{k}}{L_{X}}
$$

where $\vec{M}_{k}$ is a constant vector. Thus for $\vec{P}_{k}=0$ equation (2.18) leads to an equation only involving $X$ and $L_{X}$, namely,

$$
\frac{d^{2}}{d \eta^{2}}\left[2 a^{4} X+\frac{\vec{M}_{k}^{2}}{L_{X}^{2}}\right]+\frac{1}{L_{X}} \frac{d L_{X}}{d \eta} \frac{d}{d \eta}\left[2 a^{4} X+\frac{\vec{M}_{k}^{2}}{L_{X}^{2}}\right]-2 \frac{\vec{K}_{k}^{2}}{L_{X}^{2}}=0 .
$$

In order to solve this equation a particular lagrangian has to be chosen. Here it is assumed that the lagrangian is of the form

$$
L=-\left(\frac{X^{2}}{\Lambda^{8}}\right)^{\frac{\delta-1}{2}} X
$$

where $\delta$ is a dimensionless parameter and $\Lambda$ a dimensional constant. This is the abelian PagelsTomboulis model [21. The nonabelian theory was proposed as an effective model of low energy QCD [22]. Evidently, linear electrodynamics is recovered for the choice $\delta=1$. The lagrangian (2.21) is chosen since it leads to a simplification of the equations, but still allows to study the effects of a strongly nonlinear theory of electrodynamics on the generation of primordial magnetic fields. In general, the energy-momentum tensor derived from a lagrangian $L(X)$ is given by

$$
T_{\mu \nu}=\frac{1}{4 \pi}\left[L_{X} g^{\alpha \beta} F_{\mu \alpha} F_{\beta \nu}+g_{\mu \nu} L\right] .
$$


Furthermore, for the lagrangian (2.21) the trace of the energy-momentum tensor is given by

$$
T=\frac{1-\delta}{\pi} L
$$

which vanishes only in the case $\delta=1$ that is for linear electrodynamics. In order to check if there are any constraints on the parameter $\delta$ the energy-momentum tensor is calculated explicitly. The Maxwell tensor can be decomposed with respect to a fundamental observer with 4 -velocity $u_{\mu}$ into an electric field $\overrightarrow{\hat{E}}$ and a magnetic field $\overrightarrow{\hat{B}}$, following [23],

$$
F_{\mu \nu}=2 \hat{E}_{[\mu} u_{\nu]}-\eta_{\mu \nu \kappa \sigma} u^{\kappa} \hat{B}^{\sigma}
$$

where $\eta_{\mu \nu \kappa \sigma}=\sqrt{-g} \epsilon_{\mu \nu \kappa \sigma}$ and $u_{\mu} u^{\mu}=-1$. Then the electric and magnetic field are given, respectively, by $\hat{E}_{\mu}=F_{\nu \mu} u^{\nu}$ and $\hat{B}_{\mu}=\frac{1}{2} \eta_{\mu \nu \omega \kappa} u^{\nu} F^{\omega \kappa}$. The lab frame is defined by the proper lab coordinates $(t, \vec{r})$ determined by $d t=a d \eta, d \vec{r}=a d \vec{x}$. Applying a coordinate transformation then gives the relation between the fields measured by a fundamental observer and the lab frame. Thus using the four velocity of the fluid $u^{\mu}=\left(a^{-1}, 0,0,0\right)$ this gives the relation [24]

$$
E_{i}=a \hat{E}_{i}, \quad B_{i}=a \hat{B}_{i}
$$

As shown in 23] the energy-momentum tensor of an electromagnetic field can be cast into the form of an imperfect fluid. The energy-momentum tensor of an imperfect fluid is given by (see for example, [23]),

$$
T_{\mu \nu}=\rho u_{\mu} u_{\nu}+p h_{\mu \nu}+2 q_{(\mu} u_{\nu)}+\pi_{\mu \nu}
$$

where $\rho$ is the energy density, $p$ the pressure, $q_{\mu}$ the heat flux vector and $\pi_{\mu \nu}$ an anisotropic pressure contribution of the fluid. $h_{\mu \nu}=g_{\mu \nu}+u_{\mu} u_{\nu}$ is the metric on the space-like hypersurfaces orthogonal to $u_{\mu}$. With $q_{\mu} u^{\mu}=0$ and $\pi_{\mu \nu} u^{\mu}=0$,

$$
\begin{aligned}
\rho & =T_{\mu \nu} u^{\mu} u^{\nu} & & q_{\alpha}=-T_{\mu \nu} u^{\mu} h_{\alpha}^{\nu} \\
Q_{\alpha \beta} & \equiv T_{\mu \nu} h_{\alpha}^{\mu} h_{\beta}^{\nu} & & Q_{\alpha \beta}=p h_{\alpha \beta}+\pi_{\alpha \beta} .
\end{aligned}
$$

Thus using equations (2.22) and (2.24) the energy density and the heat flux vector for the PagelsTomboulis model (2.21) are found to be

$$
\begin{aligned}
\rho & =-\frac{1}{8 \pi} \frac{L}{X}\left[(2 \delta-1) \hat{E}_{\alpha} \hat{E}^{\alpha}+\hat{B}_{\alpha} \hat{B}^{\alpha}\right] \\
q_{\alpha} & =\frac{\delta}{4 \pi} \frac{L}{X} \eta_{\alpha \rho \xi \sigma} u^{\rho} \hat{E}^{\xi} \hat{B}^{\sigma} .
\end{aligned}
$$

Imposing the condition that $\pi_{\alpha \beta}$ is trace-free then the pressure and $\pi_{\alpha \beta}$ are given by

$$
\begin{aligned}
p & =\frac{1}{3} \rho-\frac{\delta-1}{3 \pi} L \\
\pi_{\alpha \beta} & =-\frac{\delta}{4 \pi} \frac{L}{X}\left[\frac{1}{3}\left(\hat{E}_{\mu} \hat{E}^{\mu}+\hat{B}_{\mu} \hat{B}^{\mu}\right) h_{\alpha \beta}-\left(\hat{E}_{\alpha} \hat{E}_{\beta}+\hat{B}_{\alpha} \hat{B}_{\beta}\right)\right] .
\end{aligned}
$$

Thus considering $\rho$ (cf. equation (2.28) ) in general there is a constraint on $\delta$. Namely, the positivity of $\rho$ requires $\delta \geq \frac{1}{2}$. 
Although in this work the abelian Pagels-Tomboulis model (cf. equation (2.21)) is used, for completeness, other types of lagrangians describing theories of nonlinear electrodynamics are briefly summarized in the following. The self-interaction energy of a slowly varying, but arbitrarily strong electromagnetic field was calculated by Heisenberg and Euler [10, 11]. Expanding the resulting lagrangian into an asymptotic series gives a lagrangian of the form [10]-[12]

$$
L=X+\kappa_{0} X^{2}+\kappa_{1} Y^{2}
$$

This describes the Heisenberg-Euler theory for the choice $\kappa_{0}=\frac{8 \alpha^{2}}{45 m_{e}^{4}}$ and $\kappa_{1}=\frac{14 \alpha^{2}}{45 m_{e}^{4}}$, where $\alpha$ is the fine structure constant and $m_{e}$ the electron mass. Assuming the coefficients to be general and imagining a situation where the quadratic term in $X$ is dominant, the theory can be well approximated by the Pagels-Tomboulis lagrangian.

Born-Infeld theory is another example of a theory of nonlinear electrodynamics. It was proposed as a classical, singularity-free theory of the electron [9]. The lagrangian is given by (cf. [15]-[17])

$$
L=\frac{1}{\beta}\left(1-\sqrt{1+2 \beta^{2} X-\beta^{4} Y^{2}}\right),
$$

where $\beta$ is a parameter. This type of action also appears in the description of open string states in string or M theory [15]-[17]. In this case $\beta=2 \pi \alpha^{\prime}$ with $\alpha^{\prime}$ the string tension. Considering a general parameter $\beta$ and moreover the case in which the term $\beta^{2} X$ is dominant results in a lagrangian of the Pagels-Tomboulis form. Furthermore, the parameter $\delta$ in (2.21) is given by $\delta=\frac{1}{2}$.

However, since both invariants $X$ and $Y$ appear, the resulting equations are non trivially coupled in $X$ and $Y$ which makes it difficult to find solutions in closed form. In order to study the effects of nonlinear electrodynamics in the early universe, the abelian Pagels-Tomboulis theory (cf. equation (2.21) ) will be used. This has the advantage that even though it is a strongly nonlinear theory of electrodynamics, it is still possible to find approximate solutions in certain regimes.

Therefore, in the following we will assume that the theory is determined by the abelian PagelsTomboulis lagrangian (2.21).

\section{Estimating the magnetic field strength in the Pagels-Tomboulis model}

The following model will be considered. During de Sitter inflation electrodynamics is nonlinear and described by the Pagels-Tomboulis lagrangian (2.21). This means that electrodynamics is highly nonlinear and very different from standard Maxwell electrodynamics. At the end of inflation electrodynamics becomes linear and thus the description of reheating and the subsequent radiation dominated stage is unaltered.

The scale factor during de Sitter is given by

$$
a(\eta)=a_{1}\left(\frac{\eta}{\eta_{1}}\right)^{-1}
$$

where $\eta \leq \eta_{1}<0$. The end of inflation is assumed to be at $\eta=\eta_{1}$.

Equations (2.17) and (2.18) are coupled, since $X$ depends on $\vec{E}^{2}$ and $\vec{B}^{2}$, in particular the invariant $X$ reads, $2 a^{4} X \simeq \overrightarrow{\mathcal{B}}_{k}^{2}-\overrightarrow{\mathcal{E}}_{k}^{2}$. In order to make progress three different regimes of approximation will be considered. 
i.) $\overrightarrow{\mathcal{B}}_{k}^{2} \simeq \mathcal{O}\left(\overrightarrow{\mathcal{E}}_{k}^{2}\right)$.

ii.) $\overrightarrow{\mathcal{B}}_{k}^{2} \gg \overrightarrow{\mathcal{E}}_{k}^{2}$. This implies the approximation $2 a^{4} X \simeq \overrightarrow{\mathcal{B}}_{k}^{2}$.

iii.) $\overrightarrow{\mathcal{E}}_{k}^{2} \gg \overrightarrow{\mathcal{B}}_{k}^{2}$. This implies the approximation $2 a^{4} X \simeq-\overrightarrow{\mathcal{E}}_{k}^{2}$.

As will be explained further on, following [4] it is assumed that quantum fluctuations in the electromagnetic field provide an initial magnetic and electric field. Therefore, it seems naturally to expect that initially $\overrightarrow{\mathcal{B}}_{k}^{2} \simeq \mathcal{O}\left(\overrightarrow{\mathcal{E}}_{k}^{2}\right)$. Thus the three cases mentioned above correspond to different types of evolution of the ratio $\overrightarrow{\mathcal{B}}_{k}^{2} / \overrightarrow{\mathcal{E}}_{k}^{2}$ during inflation. After the end of inflation, during the radiation era, while the electric field decays rapidly due to plasma effects, the magnetic field remains (see, e.g., [4, 25]).

\subsection{Case i.) $\overrightarrow{\mathcal{B}}_{k}^{2} \simeq \mathcal{O}\left(\overrightarrow{\mathcal{E}}_{k}^{2}\right)$}

As a further simplification it is assumed that the contribution of the constant vector $\vec{P}_{k}^{2}$ in equation (2.17) vanishes. In this case the equation (2.20) during inflation in the Pagels-Tomboulis model (2.21) can be written in the form

$$
\begin{aligned}
{\left[\left(\frac{x}{x_{1}}\right)^{-4}-\alpha_{1}(\delta-1) y^{-2 \delta+1}\right] \ddot{y}=} & -(\delta-1)\left[\alpha_{1} \delta y^{-2 \delta+1}+\left(\frac{x}{x_{1}}\right)^{-4}\right] \frac{\dot{y}^{2}}{y} \\
& +\frac{4(\delta+1)}{x_{1}}\left(\frac{x}{x_{1}}\right)^{-5} \dot{y}-\frac{20}{x_{1}^{2}}\left(\frac{x}{x_{1}}\right)^{-6} y \\
& +\alpha_{2} y^{-2(\delta-1)}
\end{aligned}
$$

where a dot denotes the derivative with repect to $x$ and $y=y(x)$. Furthermore the following definitions have been used,

$$
x=\frac{\eta}{M_{P}^{-1}}, \quad y \equiv \frac{X}{\Lambda^{4}}, \quad \alpha_{1} \equiv \frac{\hat{M}^{2}}{\hat{\Lambda}^{4} \delta^{2} a_{1}^{4}}, \quad \alpha_{2} \equiv \frac{\hat{K}^{2}}{\hat{\Lambda}^{4} \delta^{2} a_{1}^{4}}
$$

Moreover, the hatted quantities are dimensionless constants,

$$
\hat{\Lambda} \equiv \frac{\Lambda}{M_{P}}, \quad \hat{M}^{2} \equiv \frac{\vec{M}_{k}^{2}}{M_{P}^{4}}, \quad \hat{K}^{2} \equiv \frac{\vec{K}_{k}^{2}}{M_{P}^{6}}
$$

Finally, $M_{P}$ is the Planck mass. Equation (3.2) is a nonlinear differential equation and thus to find exact solutions is not trivial. Therefore, in order to proceed one further approximation will be made. It turns out that there is an approximate solution in closed form for $\delta>1$. In this case neglecting the terms involving $\left(\frac{x}{x_{1}}\right)^{\alpha}$, with the exponents $\alpha=-4,-5,-6$, yields the equation

$$
y \ddot{y}=\delta(\dot{y})^{2}+\frac{1}{1-\delta} m^{2} y^{2},
$$

where $m^{2} \equiv \frac{\alpha_{2}}{\alpha_{1}}$ which is solved by

$$
y(x)=C_{2}\left[\cosh \left(m x+(\delta-1) m C_{1}\right)\right]^{\frac{1}{1-\delta}},
$$


where $C_{1}, C_{2}$ are constants. In general, the magnetic field is given by

$$
\vec{B}_{k}^{2}=2 X+\frac{\vec{M}_{k}^{2}}{\delta^{2} a^{4}}\left(\frac{X}{\Lambda^{4}}\right)^{-2(\delta-1)} .
$$

However, for the approximate solution (3.6) the first term becomes subdominant and the magnetic field can be approximated by

$$
\vec{B}_{k}^{2} \simeq \frac{\vec{M}_{k}^{2}}{\delta^{2} a^{4}}\left(\frac{X}{\Lambda^{4}}\right)^{-2(\delta-1)} .
$$

Thus, the magnetic field at the end of inflation at the time $\eta_{1}$ can be expressed in terms of the magnetic field at the time, say $\eta_{2}$, when the comoving length scale $\lambda$ was crossing the horizon during inflation. Thus

$$
\frac{B_{k}^{2}\left(a_{1}\right)}{B_{k}^{2}\left(a_{2}\right)} \simeq e^{-4 N(\lambda)} \frac{\cosh ^{2}\left[m\left(x_{1}+(\delta-1) C_{1}\right)\right]}{\cosh ^{2}\left[m\left(x_{2}+(\delta-1) C_{1}\right)\right]},
$$

where $N(\lambda)$ is the number of e-folds before the end of inflation at which $\lambda$ left the horizon, that is, $e^{N(\lambda)}=a_{1} / a_{2}$. Furthermore, the constant $C_{1}$ is chosen such that $(\delta-1) C_{1}=-x_{2}$. Using that during de Sitter inflation, $a=a_{1}\left(\eta_{1} / \eta\right)$ and the number of e-folds, results in the magnetic energy density $\rho_{B}$ at the end of inflation,

$$
\rho_{B}\left(a_{1}\right) \simeq \rho_{B}\left(a_{2}\right) e^{-4 N(\lambda)} \cosh ^{2}\left[-m x_{1}\left(e^{N(\lambda)}-1\right)\right],
$$

where $\rho_{B}=\frac{B^{2}}{8 \pi}$. Following [4] the ratio of magnetic energy density to radiation energy density, $r$ is introduced,

$$
r \equiv \frac{\rho_{B}}{\rho_{\gamma}}
$$

In the case of linear electrodynamics, the energy density in the magnetic field and the radiation background decay both as $a^{-4}$ and thus the ratio $r$ stays invariant as the universe expands. In order to seed a galactic dynamo $r$ has to be at least $r=10^{-37}$ corresponding to a magnetic seed field at the time of galaxy formation $B_{s} \simeq 10^{-20} \mathrm{G}$. In order to seed the galactic field directly, without a galactic dynamo operating, $r$ has to be of order $r=10^{-8}$. Furthermore, we also note, that in a flat universe with a cosmological constant, these bounds can be lowered significantly. In this case, $r$ has to be at least $r=10^{-57}$ to successfully seed a galactic dynamo [26]. This implies that the magnetic field at the time of galaxy formation has to be at least of order $B_{s} \simeq 10^{-30} \mathrm{G}$. Following [4, it is assumed that the energy density stored in the mode with comoving wavelength $\lambda$ is of the order of the energy density in a thermal bath at the Gibbons-Hawking temperature of de Sitter space. This leads to $\rho_{B}\left(a_{2}\right) \simeq H^{4} \simeq\left(\frac{M^{4}}{M_{p}^{2}}\right)^{2}$, where the constant energy density during inflation is given by $M^{4}$. Finally, using that at the end of inflation the energy density in the radiation background is given by $\rho_{\gamma}=M^{2} T_{R H}^{2}$, where $T_{R H}$ is the reheat temperature, the ratio of magnetic to radiation energy density at the end of inflation at $\eta=\eta_{1}$ is found to be,

$$
r\left(a_{1}\right) \simeq\left(\frac{M}{M_{P}}\right)^{6}\left(\frac{T_{R H}}{M_{P}}\right)^{-2} e^{-4 N(\lambda)} \cosh ^{2}\left[-m x_{1}\left(e^{N(\lambda)}-1\right)\right] .
$$


Furthermore, following [4], the number of e-folds can be found as

$$
e^{N(\lambda)} \simeq 9.2 \times 10^{25}\left(\frac{\lambda}{\mathrm{Mpc}}\right)\left(\frac{M}{M_{P}}\right)^{\frac{2}{3}}\left(\frac{T_{R H}}{M_{P}}\right)^{\frac{1}{3}}
$$

where it is assumed that the scale factor today is $a_{0}=1$ and thus comoving and physical scales coincide in the present. Thus the ratio $r$ at the end of inflation is given by

$$
r\left(a_{1}\right) \simeq 10^{-104}\left(\frac{\lambda}{\mathrm{Mpc}}\right)^{-4}\left(\frac{M}{T_{R H}}\right)^{\frac{10}{3}} \cosh ^{2}\left[-9.2 \times 10^{25}\left(\frac{\lambda}{\mathrm{Mpc}}\right)\left(\frac{M}{M_{P}}\right)^{\frac{2}{3}}\left(\frac{T_{R H}}{M_{P}}\right)^{\frac{1}{3}} m x_{1}\right]
$$

Therefore, in order to achieve, a ratio of magnetic to radiation energy density at the end of inflation, which is at least some value $r_{0}$, that is $r\left(a_{1}\right) \geq r_{0}, m x_{1}$ has to satisfy,

$$
-m x_{1} \geq 10^{-26}\left(\frac{\lambda}{\mathrm{Mpc}}\right)^{-1}\left(\frac{M}{M_{P}}\right)^{-\frac{2}{3}}\left(\frac{T_{R H}}{M_{P}}\right)^{-\frac{1}{3}} \operatorname{arccosh}\left[10^{52}\left(\frac{\lambda}{\mathrm{Mpc}}\right)^{2}\left(\frac{T_{R H}}{M}\right)^{\frac{5}{3}} r_{0}^{\frac{1}{2}}\right] .
$$

Equation (3.15) only implies a constraint on $m x_{1}$ if the argument of arccosh is bigger or equal to one which can be interpreted as a bound on the reheat temperature. Assuming a galactic scale, that is $\lambda=1 \mathrm{Mpc}$ implies,

$$
10^{52}\left(\frac{T_{R H}}{M}\right)^{\frac{5}{3}} r_{0}^{\frac{1}{2}} \geq 1
$$

which for $r_{0}=10^{-37}$ implies $T_{R H} \geq 1 \mathrm{MeV}$, where it was assumed [4] that the inflationary energy scale is given by $M=10^{17} \mathrm{GeV}$. This is always satisfied since the reheat temperature has to be at least $10 \mathrm{MeV}$ (for even lower values, see [27]) in order to allow for nucleosynthesis to take place unaltered. For a smaller value of $r_{0}$, say $r_{0}=10^{-57}$, the reheat temperature is required to be at least of order $10^{3} \mathrm{GeV}$. However, in this work the reheat temperature is assumed to be at least $T_{R H} \geq 10^{9} \mathrm{GeV}$. There is also an upper bound on $-m x_{1}$ coming from the requirement that $r<1$ in order not to overclose the universe. This implies,

$$
-m x_{1}<10^{-26}\left(\frac{\lambda}{\mathrm{Mpc}}\right)^{-1}\left(\frac{M}{M_{P}}\right)^{-\frac{2}{3}}\left(\frac{T_{R H}}{M_{P}}\right)^{-\frac{1}{3}} \operatorname{arccosh}\left[10^{52}\left(\frac{\lambda}{\mathrm{Mpc}}\right)^{2}\left(\frac{T_{R H}}{M}\right)^{\frac{5}{3}}\right] .
$$

The constraint equations (3.15) and (3.17) can always be satisfied, since, by assumption, $r_{0} \leq 1$ and, moreover, for physically interesting models $r_{0} \ll 1$. Thus assuming $\lambda=1 \mathrm{Mpc}$ and $M=10^{17}$ $\mathrm{GeV}$, the following values for $-m x_{1}$ are found. For a model with reheat temperature $T_{R H}=10^{9}$ $\mathrm{GeV}$ [4] the parameter $-m x_{1}$ has to be in the range $2.7 \times 10^{-20}<-m x_{1}<5 \times 10^{-20}$ in order to achieve a magnetic seed field with a field strength to be at least $B_{s} \simeq 10^{-20} \mathrm{G}$, corresponding to $r_{0}=10^{-37}$. For a higher reheat temperature $T_{R H}=10^{17} \mathrm{GeV}$ [4], for the same magnetic seed field strength $-m x_{1}$ has to be in the range, $9.5 \times 10^{-23}<-m x_{1}<1.5 \times 10^{-22}$. And similarly, for the less conservative bound $r_{0}=10^{-57}$, for $T_{R H}=10^{9} \mathrm{GeV},-m x_{1}$ has to be in the range $1.4 \times 10^{-20}<$ $-m x_{1}<5 \times 10^{-20}$ and for $T_{R H}=10^{17} \mathrm{GeV}$ it is found that $6.7 \times 10^{-23}<-m x_{1}<1.5 \times 10^{-22}$.

Thus there is a range of parameters for which strong enough magnetic seed fields can be created in the Pagels-Tomboulis model of nonlinear electrodynamics. Since the analysis is based on the approximate exact solution given by equation (3.6) it is also important to check that the solution 
provides a good approximation to the solution of the full differential equation (3.2). This has been done in Appendix A.

In summary, for $\delta>1$, there is an approximate analytical solution which allows to find an expression for the ratio of the magnetic to radiation energy density. There is a range of parameters for which magnetic seed fields of cosmologically interesting field strengths can be created.

\subsection{Case ii.) $\overrightarrow{\mathcal{B}}_{k}^{2} \gg \overrightarrow{\mathcal{E}}_{k}^{2}$}

In this case equation (2.18) takes the form,

$$
\frac{d^{2}}{d \eta^{2}}\left(a^{4} X\right)+(\delta-1) \frac{1}{X} \frac{d X}{d \eta} \frac{d}{d \eta}\left(a^{4} X\right)-\frac{\vec{K}_{k}^{2}}{\delta^{2}}\left(\frac{X}{\Lambda^{4}}\right)^{-2(\delta-1)}=0,
$$

where it has been used that $2 X \simeq \vec{B}_{k}^{2}$. It is possible to find different types of solutions of equation (3.18) depending on the value of the parameter $\delta$ of the Pagels-Tomboulis model. On the one hand there are power law solutions for $\delta \neq \frac{1}{2}$ and $\delta \neq \frac{5}{4}$. On the other hand there are solutions with a distinct behaviour for $\delta=\frac{1}{2}$ and $\delta=\frac{5}{4}$. Actually of the latter ones only the case $\delta=\frac{1}{2}$ will be discussed explicitly. This is so because for $\delta=\frac{5}{4}$ it is only possible to find an implicit solution depending on Euler's $\beta$ function which makes it very difficult to estimate the primordial magnetic field strength.

\subsubsection{Solution for $\delta \neq \frac{1}{2}$ and $\delta \neq \frac{5}{4}$}

For $\delta \neq \frac{1}{2}$ and $\delta \neq \frac{5}{4}$, equation (3.18) can be solved by a power-law function,

$$
X=X_{1}\left(\frac{\eta}{\eta_{1}}\right)^{\alpha} .
$$

This leads to the solution for the magnetic field

$$
\vec{B}_{k}^{2}=2 \Lambda^{4}\left[\frac{2}{\alpha_{2} x_{1}^{2}}\left(\frac{5-4 \delta}{2 \delta-1}\right)^{2}\right]^{\frac{1}{1-2 \delta}}\left(\frac{\eta}{\eta_{1}}\right)^{\frac{6}{2 \delta-1}}
$$

where, as before, $\alpha_{2} \equiv \frac{\hat{K}^{2}}{a_{1}^{4} \hat{\Lambda}^{4} \delta^{2}}$. Thus using the definitions as given for case i.) (cf. section 3.1) the ratio of magnetic energy density to radiation energy density at the end of inflation $r\left(a_{1}\right)$ is found to be, for $\delta \neq \frac{1}{2}, \delta \neq \frac{5}{4}$,

$$
r\left(a_{1}\right) \simeq\left(9.2 \times 10^{25}\right)^{-\frac{6}{2 \delta-1}}\left(\frac{\lambda}{\mathrm{Mpc}}\right)^{-\frac{6}{2 \delta-1}}\left(\frac{M}{M_{P}}\right)^{2 \frac{6 \delta-5}{2 \delta-1}}\left(\frac{T_{R H}}{M_{P}}\right)^{-\frac{4 \delta}{2 \delta-1}}
$$

The range of validity of the assumption $\overrightarrow{\mathcal{B}}_{k}^{2} \gg \overrightarrow{\mathcal{E}}_{k}^{2}$ can be checked to first order by using the solution for $B_{k}^{2} \simeq 2 X$, (cf. equation (3.20) $)$ in the equation for $\overrightarrow{\mathcal{E}}_{k}^{2}$, equation (2.17). This leads to

$$
\overrightarrow{\mathcal{E}}_{k}^{2 \prime \prime}+\beta_{1} \eta^{-1} \overrightarrow{\mathcal{E}}_{k}^{2 \prime}+\beta_{2} \eta^{-2} \overrightarrow{\mathcal{E}}_{k}^{2}=2 \vec{P}_{k}^{2},
$$

where $\beta_{1} \equiv 3 \alpha(\delta-1)$ and $\beta_{2} \equiv 2 \alpha(\delta-1)[\alpha(\delta-1)-1]$. This equation is solved by, for $\vec{P}_{k}^{2} \neq 0$,

$$
\overrightarrow{\mathcal{E}}_{k}^{2}=\vec{P}_{k}^{2}\left(\frac{2 \delta-1}{8 \delta-7}\right)^{2} \eta^{2}+c_{0}\left(\frac{\eta}{\eta_{1}}\right)^{\frac{-4 \delta+5}{2 \delta-1}}+c_{1}\left(\frac{\eta}{\eta_{1}}\right)^{-\frac{12(\delta-1)}{2 \delta-1}}
$$


where $c_{0}$ and $c_{1}$ are constants. For $\vec{P}_{k}^{2}=0$, the solution is given by equation (2.19) which will be discussed below. During de Sitter inflation the scale factor is given by equation (3.1). Thus, finally, the ratio $\overrightarrow{\mathcal{E}}_{k}^{2} / \overrightarrow{\mathcal{B}}_{k}^{2}$ is given by

$$
\frac{\overrightarrow{\mathcal{E}}_{k}^{2}}{\overrightarrow{\mathcal{B}}_{k}^{2}} \simeq \mu_{0}\left(\frac{\eta}{\eta_{1}}\right)^{12 \frac{\delta-1}{2 \delta-1}}+\mu_{1}\left(\frac{\eta}{\eta_{1}}\right)^{\frac{4 \delta-5}{2 \delta-1}}+\mu_{2}\left(\frac{\eta}{\eta_{1}}\right)^{-2}
$$

where $\mu_{0}, \mu_{1}$ and $\mu_{2}$ are constants depending on the constants of the solutions of the electric and magnetic field. However, their explicit form is not important here. Since during inflation, $\eta<\eta_{1}<0$, and hence $\eta / \eta_{1}>1$. At $\eta=\eta_{2}$, that is at the time when the mode is leaving the horizon during inflation, the initial condition, $\frac{\overrightarrow{\mathcal{E}}_{k}^{2}}{\overrightarrow{\mathcal{B}}_{k}^{2}}\left(\eta_{2}\right)=1$ is imposed. In order for the approximation to be consistent, it is required that the solution evolves such that $\frac{\overrightarrow{\mathcal{E}}_{k}^{2}}{\overline{\mathcal{B}}_{k}^{2}} \leq 1$. So assuming that each of the terms is of the order of $\frac{1}{3}$ at $\eta=\eta_{2}$ then the constants $\mu_{0}, \mu_{1}$ and $\mu_{2}$ can be estimated in terms of $\frac{\eta_{2}}{\eta_{1}}$. This leads to

$$
\frac{\overrightarrow{\mathcal{E}}_{k}^{2}}{\overrightarrow{\mathcal{B}}_{k}^{2}}=\frac{1}{3}\left(\frac{\eta}{\eta_{2}}\right)^{12 \frac{\delta-1}{2 \delta-1}}+\frac{1}{3}\left(\frac{\eta}{\eta_{2}}\right)^{\frac{4 \delta-5}{2 \delta-1}}+\frac{1}{3}\left(\frac{\eta}{\eta_{2}}\right)^{-2}
$$

Thus the last term is growing and in general, $\frac{\overrightarrow{\mathcal{E}}_{k}^{2}}{\overrightarrow{\mathcal{B}}_{k}^{2}}$ is not smaller than one. Thus in order for the solution to be consistent within the approximation, the constant $c_{1}$ in equation (3.23) has to be set to zero. Furthermore, the exponents in equation (3.25) have to be positive, imposing the constraints, $\delta<\frac{1}{2}$ or $\delta>\frac{5}{4}$. The former one is ruled out since $\delta \geq \frac{1}{2}$. Then by assuming that the two remaining terms, after setting $c_{1}$ to zero, contribute equally at $\eta=\eta_{2}$, the ratio $\frac{\overrightarrow{\mathcal{E}}_{k}^{2}}{\overline{\mathcal{B}}_{k}^{2}} \leq 1$ for $\eta \geq \eta_{2}$ and $\delta>\frac{5}{4}$.

In order to seed the galactic dynamo, it is required that $r \geq r_{0}$ where $r_{0}$ is the lower bound on the strength of the magnetic field. In the expression for $r=\frac{\rho_{B}}{\rho_{\gamma}}$ at the end of inflation there are, apart from $\delta$, two parameters: on the one hand the constant energy density during inflation given by $M^{4}$ and on the other hand the reheat temperature, $T_{R H}$. Following [4] $M$ is chosen to be $M=10^{17} \mathrm{GeV}$. The reheat temperature depends on the details of the reheating process. It can be as low as $4 \mathrm{MeV}$ [27] and in general, one expects an upper limit $T_{R H} \leq M$. However, in supersymmetric theories this limit is lowered down to $10^{9} \mathrm{GeV}$ [28].

In Figure $1 \log r$ is plotted against the Pagels-Tomboulis parameter $\delta$ for the inflationary energy scale $M=10^{17} \mathrm{GeV}$ for the reheat temperatures $T_{R H}=10^{17} \mathrm{GeV}$ and $T_{R H}=10^{9} \mathrm{GeV}$. As can be seen from Figure 1 for $\delta>\frac{5}{4}$ there is range of $\delta$ for which primordial magnetic fields with cosmologically interesting field strengths can be generated.

In the case $\vec{P}_{k}^{2}=0$ the solution for $\overrightarrow{\mathcal{E}}_{k}$ is given by equation (2.19). This leads to

$$
\overrightarrow{\mathcal{E}}_{k}^{2} \simeq \frac{\vec{M}_{k}^{2}}{\delta^{2}}\left(\frac{X^{2}}{\Lambda^{8}}\right)^{1-\delta}
$$

and thus

$$
\frac{\overrightarrow{\mathcal{E}}_{k}^{2}}{\overrightarrow{\mathcal{B}}_{k}^{2}} \simeq \frac{1}{m^{2} x_{1}^{2}}\left(\frac{5-4 \delta}{2 \delta-1}\right)^{2}\left(\frac{\eta}{\eta_{1}}\right)^{-2}
$$




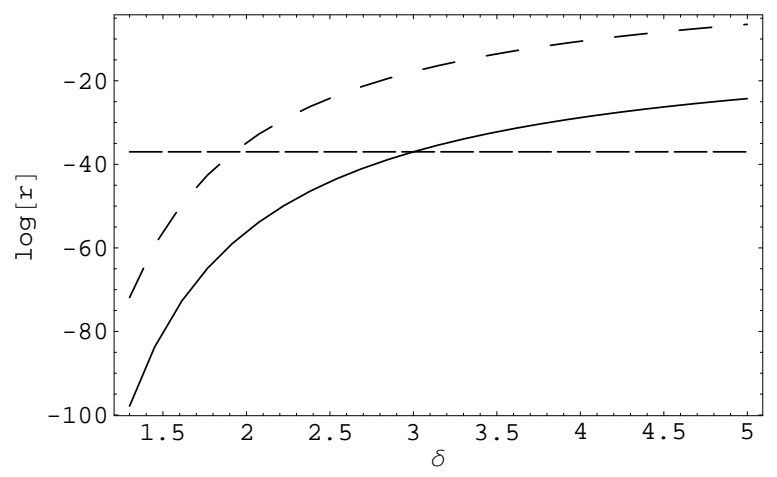

Figure 1: For $\lambda=1 \mathrm{Mpc} \log r$ (cf. equation (3.21)) is shown as a function of $\delta$ for $T_{R H}=10^{17} \mathrm{GeV}$ (black line) and $T_{R H}=10^{9} \mathrm{GeV}$ (long-dashed line). The dashed line corresponds to $r=10^{-37}$.

where as before (cf. equation (3.2) $) m^{2}=\frac{\alpha_{2}}{\alpha_{1}}$. Thus imposing the initial condition $\frac{\overrightarrow{\mathcal{E}}_{k}^{2}}{\overline{\mathcal{B}}_{k}^{2}}\left(\eta_{2}\right) \simeq 1$ implies

$$
\frac{\overrightarrow{\mathcal{E}}_{k}^{2}}{\overrightarrow{\mathcal{B}}_{k}^{2}}=\left(\frac{\eta_{2}}{\eta}\right)^{2}
$$

which implies $\frac{\overrightarrow{\mathcal{E}}_{k}^{2}}{\overrightarrow{\mathcal{B}}_{k}^{2}} \geq 1$ for $\eta \geq \eta_{2}$. Thus this solution is not consistent with the approximation.

\subsubsection{Solution for $\delta=\frac{1}{2}$}

In this case equation (3.18) can be written as

$$
\ddot{g}-\frac{1}{2}\left(\frac{4}{x}+\frac{\dot{g}}{g}\right) \dot{g}-\alpha_{2}\left(\frac{x}{x_{1}}\right)^{4} g=0,
$$

where $g \equiv\left(\frac{x}{x_{1}}\right)^{-4} X, x \equiv \eta / M_{P}^{-1}$ and $\alpha_{2}$ a constant as defined before, (cf. equation (3.3)). This is solved by $g(x)=c_{2} \cosh ^{2}\left[\left(\frac{\alpha_{2}}{18}\right)^{\frac{1}{2}} x_{1}\left(\frac{x}{x_{1}}\right)^{3}+c_{1}\right]$, where $c_{1}$ and $c_{2}$ are constants. Thus with $\vec{B}_{k}^{2} \simeq 2 X$ it follows that

$$
\vec{B}_{k}^{2} \simeq 2 c_{2}\left(\frac{x}{x_{1}}\right)^{4} \cosh ^{2}\left[\left(\frac{\alpha_{2}}{18}\right)^{\frac{1}{2}} x_{1}\left(\frac{x}{x_{1}}\right)^{3}+c_{1}\right] .
$$

This then leads to the ratio of magnetic energy density to radiation background energy density $r=\frac{\rho_{B}}{\rho_{\gamma}}$ at the end of inflation,

$$
r\left(a_{1}\right) \simeq 10^{-104}\left(\frac{\lambda}{\mathrm{Mpc}}\right)^{-4}\left(\frac{M}{T_{R H}}\right)^{\frac{10}{3}} \cosh ^{2}\left[-8 \times 10^{77} x_{1}\left(\frac{\alpha_{2}}{18}\right)^{\frac{1}{2}}\left(\frac{\lambda}{\mathrm{Mpc}}\right)^{3}\left(\frac{M}{M_{P}}\right)^{2} \frac{T_{R H}}{M_{P}}\right] .
$$


Here the constant $c_{1}$ has been chosen as $c_{1} \equiv-\left(\frac{\alpha_{2}}{18}\right)^{\frac{1}{2}} x_{1}\left(\frac{\eta_{2}}{\eta_{1}}\right)^{3}$. Imposing the condition that $r_{0}<r\left(a_{1}\right)<1$ results, for $\lambda=1 \mathrm{Mpc}$, in

$$
\begin{aligned}
10^{-78}\left(\frac{M}{M_{P}}\right)^{-2} & \left(\frac{T_{R H}}{M_{P}}\right)^{-1} \operatorname{arccosh}\left[10^{52}\left(\frac{T_{R H}}{M}\right)^{\frac{5}{3}} r_{0}^{\frac{1}{2}}\right]<-x_{1}\left(\frac{\alpha_{2}}{18}\right)^{\frac{1}{2}} \\
& <10^{-78}\left(\frac{M}{M_{P}}\right)^{-2}\left(\frac{T_{R H}}{M_{P}}\right)^{-1} \operatorname{arccosh}\left[10^{52}\left(\frac{T_{R H}}{M}\right)^{\frac{5}{3}}\right] .
\end{aligned}
$$

The resulting values for different choices of $T_{R H}, M$ and $r_{0}$ are given in Table 1 . Furthermore,

\begin{tabular}{|c|c|c||c|c|}
\hline$T_{R H}(\mathrm{GeV})$ & $M(\mathrm{GeV})$ & $r_{0}$ & $-x_{1}\left(\frac{\alpha_{2}}{18}\right)_{\text {low }}^{\frac{1}{2}}$ & $-x_{1}\left(\frac{\alpha_{2}}{18}\right)^{\frac{1}{2}}$ \\
\hline \hline $10^{9}$ & $10^{17}$ & $10^{-37}$ & $8.6 \times 10^{-63}$ & $1.6 \times 10^{-62}$ \\
\hline $10^{9}$ & $10^{17}$ & $10^{-57}$ & $4.4 \times 10^{-63}$ & $1.6 \times 10^{-62}$ \\
\hline $10^{17}$ & $10^{17}$ & $10^{-37}$ & $8.6 \times 10^{-71}$ & $1.6 \times 10^{-70}$ \\
\hline $10^{17}$ & $10^{17}$ & $10^{-57}$ & $4.4 \times 10^{-71}$ & $1.6 \times 10^{-70}$ \\
\hline
\end{tabular}

Table 1: Lower and upper bounds of $-x_{1}\left(\frac{\alpha_{2}}{18}\right)^{\frac{1}{2}}$ derived from equation (3.32) for different values of the reheat temperature $T_{R H}$, the constant energy density during inflation determined by $M$ and the lower limit on the field strength of a primordial magnetic seed field determined by $r_{0}$. The notation used indicates $-x_{1}\left(\frac{\alpha_{2}}{18}\right)_{\text {low }}^{\frac{1}{2}}<-x_{1}\left(\frac{\alpha_{2}}{18}\right)^{\frac{1}{2}}<-x_{1}\left(\frac{\alpha_{2}}{18}\right)_{\text {up }}^{\frac{1}{2}}$.

in order to check the validity of the solution (3.30) which was derived under the assumption that $\overrightarrow{\mathcal{E}}_{k}^{2} / \overrightarrow{\mathcal{B}}_{k}^{2} \ll 1$, we consider the cases $\vec{P}_{k}^{2}>0$ and $\vec{P}_{k}^{2}=0$. In the case $\overrightarrow{P_{k}^{2}}>0$ the electric field strength is determined by equation (2.17). As a first approximation, the solution for $X \simeq \frac{1}{2} \vec{B}_{k}^{2}$, where $\vec{B}_{k}^{2}$ is given by (3.30), will be used in (2.17). For consistency, the resulting solution for the electric field strength should be much smaller than the magnetic field strength. In equation (2.17) the expressions for $\frac{L_{X}^{\prime}}{L_{X}}$ and $\frac{L_{X}^{\prime \prime}}{L_{X}}$ are needed which are given in Appendix B. The cosmologically interesting values of $\mu \equiv-\left(\frac{\alpha_{2}}{18}\right)^{\frac{1}{2}} x_{1}$ are very small, $\mu \lesssim \mathcal{O}\left(10^{-62}\right)$, as can be seen from Table 1 . Thus to zeroth order in $\mu$ equation (2.17) becomes,

$$
\overrightarrow{\mathcal{E}}_{k}^{2 \prime \prime}-\frac{6}{\eta} \overrightarrow{\mathcal{E}}_{k}^{2 \prime}+\frac{12}{\eta^{2}} \overrightarrow{\mathcal{E}}_{k}^{2}=2 \vec{P}_{k}^{2}
$$

which is solved by

$$
\overrightarrow{\mathcal{E}}_{k}^{2}=\vec{P}_{k}^{2} \eta_{1}^{2}\left(\frac{\eta}{\eta_{1}}\right)^{2}+\beta_{0}\left(\frac{\eta}{\eta_{1}}\right)^{3}+\beta_{1}\left(\frac{\eta}{\eta_{1}}\right)^{4},
$$

where $\beta_{0}$ and $\beta_{1}$ are constants. Therefore

$$
\frac{\overrightarrow{\mathcal{E}}_{k}^{2}}{\overrightarrow{\mathcal{B}}_{k}^{2}} \simeq \frac{\vec{P}_{k}^{2} \eta_{1}^{2}\left(\frac{\eta}{\eta_{1}}\right)^{2}+\beta_{0}\left(\frac{\eta}{\eta_{1}}\right)^{3}+\beta_{1}\left(\frac{\eta}{\eta_{1}}\right)^{4}}{2 c_{2} a_{1}^{4} \cosh ^{2}\left[-\left(\frac{\alpha_{2}}{18}\right)^{\frac{1}{2}} x_{1}\left[\left(\frac{\eta_{2}}{\eta_{1}}\right)^{3}-\left(\frac{\eta}{\eta_{1}}\right)^{3}\right]\right]} .
$$


Imposing the initial condition $\frac{\overrightarrow{\mathcal{E}}_{k}^{2}}{\overrightarrow{\mathcal{B}}_{k}^{2}}\left(\eta_{2}\right)=1$ it can be seen that $\frac{\overrightarrow{\mathcal{E}}_{k}^{2}}{\overrightarrow{\mathcal{B}}_{k}^{2}}$ is decreasing very fast and thus the solution (3.30) is consistent at lowest order in $\mu$.

In the case $\vec{P}_{k}^{2}=0$ the solution for the electric field strength is given by equation (2.19), leading to

$$
\overrightarrow{\mathcal{E}}_{k}^{2} \simeq 4 \vec{M}_{k}^{2}\left(\frac{X^{2}}{\Lambda^{8}}\right)^{\frac{1}{2}}
$$

This implies

$$
\frac{\overrightarrow{\mathcal{E}}_{k}^{2}}{\overrightarrow{\mathcal{B}}_{k}^{2}} \simeq \frac{\alpha_{1}}{2}\left(\frac{\eta}{\eta_{1}}\right)^{4}
$$

Imposing that initially $\frac{\overrightarrow{\mathcal{E}}_{k}^{2}}{\overline{\mathcal{B}}_{k}^{2}}\left(\eta_{2}\right)=1$ it follows that

$$
\frac{\overrightarrow{\mathcal{E}}_{k}^{2}}{\overrightarrow{\mathcal{B}}_{k}^{2}}=\left(\frac{\eta}{\eta_{2}}\right)^{4}
$$

which is smaller than one for $\eta>\eta_{2}$. Hence the solution is consistent with the approximation.

Thus, for the solution in this case, there is a choice of parameters which allows to create primordial magnetic fields of cosmologically interesting field strengths. This holds for both cases, $\vec{P}^{2}>0$ and $\vec{P}^{2}=0$.

\subsection{Case iii.) $\overrightarrow{\mathcal{E}}_{k}^{2} \gg \overrightarrow{\mathcal{B}}_{k}^{2}$}

Starting out at the same order of magnitude, in this approximation at the end of inflation the energy density in the electric field is much larger than in the magnetic field. The approximation implies that $\overrightarrow{\mathcal{E}}_{k}^{2} \simeq-2 a^{4} X$. In the case $\vec{P}_{k}^{2}>0$ equation (2.17) yields to

$$
\frac{d^{2}}{d \eta^{2}}\left(a^{4} X\right)+3(\delta-1) \frac{X^{\prime}}{X} \frac{d}{d \eta}\left(a^{4} X\right)+2(\delta-1)\left[\frac{X^{\prime \prime}}{X}+(\delta-2)\left(\frac{X^{\prime}}{X}\right)^{2}\right] a^{4} X=-\vec{P}_{k}^{2},
$$

which is solved by, for $\delta \neq \frac{5}{6}$,

$$
X=-\frac{\vec{P}_{k}^{2} \eta_{1}^{2}}{2 a_{1}^{4}(6 \delta-5)^{2}}\left(\frac{\eta}{\eta_{1}}\right)^{6}
$$

Thus using $X=X_{1}\left(\frac{\eta}{\eta_{1}}\right)^{\alpha}$ in the equation determining the magnetic field (cf. equation (2.18)) gives,

$$
\vec{B}_{k}^{2}=\frac{\vec{K}_{k}^{2} \eta_{1}^{2}}{a_{1}^{4} \delta^{2}[1-\alpha(\delta-1)]^{2}}\left(\frac{X_{1}^{2}}{\Lambda^{8}}\right)^{-(\delta-1)}\left(\frac{\eta}{\eta_{1}}\right)^{6-2 \alpha(\delta-1)}+b_{0}\left(\frac{\eta}{\eta_{1}}\right)^{4}+b_{1}\left(\frac{\eta}{\eta_{1}}\right)^{5-\alpha(\delta-1)}
$$

where $b_{0}$ and $b_{1}$ are constants and $X_{1}=-\frac{\vec{P}_{k}^{2} \eta_{1}^{2}}{2 a_{1}^{4}(6 \delta-5)^{2}}$. With $\alpha=6$ this leads to

$$
\frac{B_{k}^{2}}{E_{k}^{2}} \simeq \mu_{0}\left(\frac{\eta}{\eta_{1}}\right)^{-12(\delta-1)}+\mu_{1}\left(\frac{\eta}{\eta_{1}}\right)^{-2}+\mu_{2}\left(\frac{\eta}{\eta_{1}}\right)^{5-6 \delta}
$$


where $\mu_{0}, \mu_{1}$ and $\mu_{2}$ are constants which can be found from the expressions for $E_{k}^{2}$ and $B_{k}^{2}$. Imposing the initial condition $E_{k}^{2}\left(\eta_{2}\right) \simeq B_{k}^{2}\left(\eta_{2}\right)$ and that all terms contribute equally at this time results in

$$
\frac{B_{k}^{2}}{E_{k}^{2}} \simeq \frac{1}{3}\left(\frac{\eta_{2}}{\eta}\right)^{12(\delta-1)}+\frac{1}{3}\left(\frac{\eta_{2}}{\eta}\right)^{2}+\frac{1}{3}\left(\frac{\eta_{2}}{\eta}\right)^{6 \delta-5} .
$$

Thus in order to achieve, $B_{k}^{2} / E_{k}^{2} \leq 1$ the constant $b_{0}$ in equation (3.41) has to be set to zero. With the remaining two terms contributing equally at $\eta=\eta_{2}$ and requiring $\frac{1}{2}<\delta<\frac{5}{6}$ leads to solutions which are consistent with the assumption $B_{k}^{2} / E_{k}^{2} \leq 1$. Furthermore, in the expression for $B_{k}^{2}$ the dominant contribution comes from the last term, thus the evolution of the magnetic field is given by $\vec{B}_{k}^{2} \sim\left(\frac{\eta}{\eta_{1}}\right)^{\beta}$ where $\beta=11-6 \delta$ and $\frac{1}{2}<\delta<\frac{5}{6}$. Moreover, the ratio of the energy density in the magnetic field and the background radiation $r$ at the end of inflation can be calculated, resulting in

$$
r\left(a_{1}\right) \simeq\left(9.2 \times 10^{25}\right)^{-\beta}\left(\frac{\lambda}{\mathrm{Mpc}}\right)^{-\beta}\left(\frac{M}{M_{P}}\right)^{6-\frac{2 \beta}{3}}\left(\frac{T_{R H}}{M_{P}}\right)^{-2-\frac{\beta}{3}} .
$$

In Figure $2 \log r$ is shown. As can be seen the resulting magnetic field strengths are far below the lower boundary of $r_{0}=10^{-37}$, corresponding to a magnetic seed field of $B_{s}=10^{-20} \mathrm{G}$.
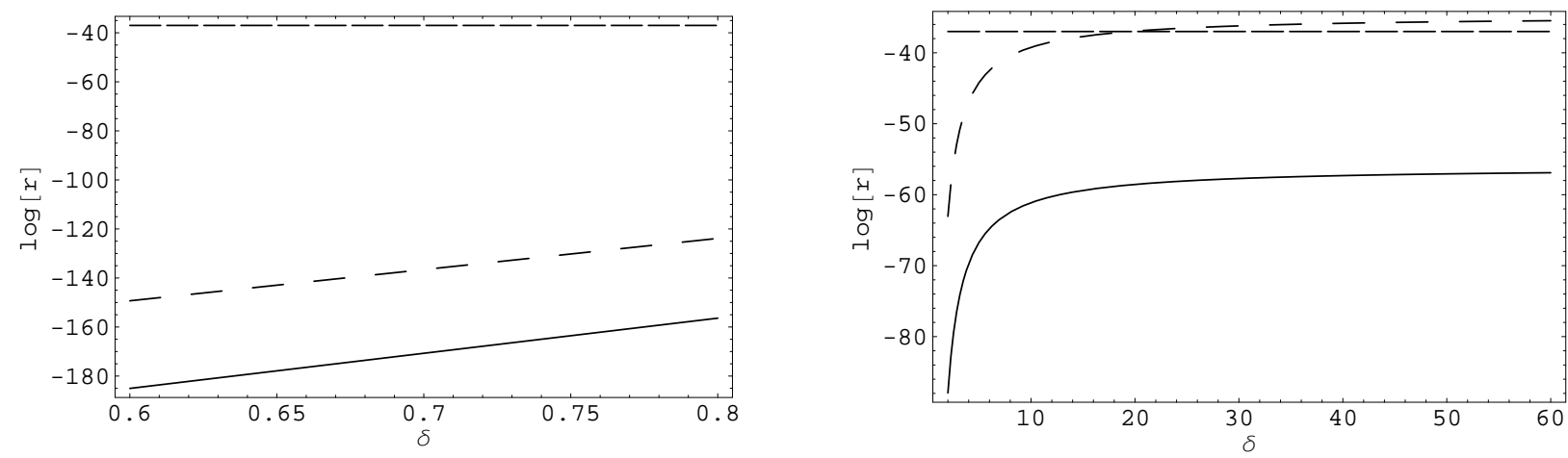

Figure 2: For $\lambda=1 \mathrm{Mpc} \log r$ (cf. equation (3.44)) is shown as a function of $\delta$ for $T_{R H}=10^{17} \mathrm{GeV}$ (black line) and $T_{R H}=10^{9} \mathrm{GeV}$ (long-dashed line). The dashed line corresponds to $r=10^{-37}$. The left panel corresponds to the case $\vec{P}_{k}^{2}>0$. The right panel corresponds to the case $\vec{P}_{k}^{2}=0$.

Finally, the solution for $\vec{P}_{k}^{2} \equiv 0$ will be discussed. Thus using equation (2.19) and $X \simeq-\frac{1}{2} \vec{E}_{k}^{2}$ yields to

$$
\vec{E}_{k}^{2} \sim\left(\frac{\eta}{\eta_{1}}\right)^{\frac{4}{2 \delta-1}}
$$

It is found that the solutions are consistent with the assumption $\overrightarrow{\mathcal{E}}_{k}^{2}>\overrightarrow{\mathcal{B}}_{k}^{2}$ for $1<\delta<\frac{3}{2}$ implying $\beta=4$ and for $\delta>\frac{3}{2}$ corresponding to $\beta=2 \frac{2 \delta+1}{2 \delta-1}$. Thus using $\beta=4$ and $\lambda=1 \mathrm{Mpc}$ yields to $r\left(a_{1}\right) \simeq 10^{-104}$ for $M=10^{17} \mathrm{GeV}$ and $T_{R H}=10^{17} \mathrm{GeV}$. Moreover $r\left(a_{1}\right) \simeq 10^{-77}$ is found for $M=10^{17} \mathrm{GeV}$ and $T_{R H}=10^{9} \mathrm{GeV}$. These values are far below the lower bounds on the primordial magnetic field required to seed the galactic field. The results for $\delta>\frac{3}{2}$ are shown in Figure 2. As can be seen for $T_{R H}=10^{9} \mathrm{GeV}$ magnetic fields satisfying $r>10^{-37}$ can be generated for $\delta>19.5$. 


\subsection{Discussion}

Solutions for the magnetic energy density of nonlinear electrodynamics with a lagrangian given by $L=-\left(\frac{X^{2}}{\Lambda^{8}}\right)^{\frac{\delta-1}{2}} X$, where $\Lambda$ and $\delta$ are constant parameters have been found for different approximations. The solutions are determined by the system of equations (2.17) and (2.18). These equations depend on two constants, $\vec{P}_{k}^{2}$ and $\vec{K}_{k}^{2}$. In the case where $\vec{P}_{k}^{2}=0$, equation (2.17) is replaced by equation (2.19) which involves a new constant, $\vec{M}_{k}^{2}$, in the final equation (2.20). Furthermore, $\vec{M}_{k}^{2}$ and $\vec{K}_{k}^{2}$ lead to the definitions of the two dimensionless constants $\alpha_{1}$ and $\alpha_{2}$ in equation (3.2) and $m^{2} \equiv \frac{\alpha_{2}}{\alpha_{1}}$.

It is assumed that the electric and magnetic fields, respectively, have their origin in quantum fluctuations during inflation. Therefore, it seems quite natural to impose that initially, that is at the time when the perturbation leaves the horizon, the energy density in the electric and magnetic field are of the same order. During the later evolution these quantites of course can be very different. In order to solve the equations, we have assumed three different types of evolution of the ratio of the energy densities in the electric and magnetic field. This has led to estimates of the primordial magnetic field at the time of galaxy formation.

In the case $\overrightarrow{\mathcal{B}}_{k}^{2} \simeq \mathcal{O}\left(\overrightarrow{\mathcal{E}}_{k}^{2}\right)$ for $\vec{P}_{k}^{2}=0$ it was found that strong primordial magnetic fields can be generated.

Assuming that during inflation $\overrightarrow{\mathcal{B}}_{k}^{2} \gg \overrightarrow{\mathcal{E}}_{k}^{2}$ there is a range of the Pagels-Tomboulis parameter $\delta$ for which in the case $\vec{P}_{k}^{2}>0$ primordial magnetic fields can be generated that are strong enough to seed the galactic dynamo. In particular, for $\delta>1.9$ for $T_{R H}=10^{17} \mathrm{GeV}$ and for $\delta>3.0$ for $T_{R H}=10^{9} \mathrm{GeV}$ the ratio of the energy density of the magnetic field over the energy density of the background radiation $\mathrm{r}$ is found to be $r>10^{-37}$ corresponding to a primordial magnetic field of at least $B_{s}=10^{-20} \mathrm{G}$ (cf. Figure 1). However, in the case $\vec{P}^{2}=0$ this solution is not consistent with the approximation $\overrightarrow{\mathcal{B}}_{k}^{2} \gg \overrightarrow{\mathcal{E}}_{k}^{2}$. Thus it cannot be used to estimate the primordial magnetic field in this case. The former class of solutions do not inlude the case $\delta=\frac{1}{2}$. In that case the solutions found for the electric and magnetic field are consistent with the approximation for $\vec{P}_{k}^{2}>0$ and $\vec{P}_{k}^{2}=0$. Moreover, the resulting magnetic field is strong enough to seed the galactic dynamo.

Finally, making the approximation $\overrightarrow{\mathcal{E}}_{k}^{2} \gg \overrightarrow{\mathcal{B}}_{k}^{2}$ yields in the case $\vec{P}_{k}^{2}>0$ to very weak magnetic fields. However, in the case $\vec{P}_{k}^{2}=0$, for $\delta>19.5$ and a reheat temperature $T_{R H}=10^{9} \mathrm{GeV}$ primordial magnetic fields result which could successfully act as seed fields for the galactic dynamo (cf. Figure 2).

\section{Conclusions}

Observations of magnetic fields on large scales provide an intriguing problem. A possible class of mechanisms to create such fields is provided by inflationary models. Fluctuations in the electromagnetic field are amplified during inflation and provide a seed magnetic field at the time of structure formation which might be further amplified by a dynamo process. In general a sufficiently strong initial field strength can only be achieved if the conformal invariance of electrodynamics is broken. This has been realized, for example, in models where the Maxwell lagrangian has been coupled to a scalar field, to curvature terms, etc. or by breaking Lorentz invariance or adding extra dimensions.

Here nonlinear electrodynamics has been considered. It has been assumed that whereas dur- 
ing the early universe electrodynamics is nonlinear it becomes linear at the end of inflation. In particular the evolution of the magnetic energy density has been studied in a model of nonlinear

electrodynamics which is described by a lagrangian of the form $L \sim-\left[\left(F_{\mu \nu} F^{\mu \nu}\right)^{2} / \Lambda^{8}\right]^{\frac{\delta-1}{2}} F_{\mu \nu} F^{\mu \nu}$, where $\Lambda$ and $\delta$ are parameters. Originally the nonabelian version of this model had been proposed to describe low energy QCD [22]. Here this model has been chosen as it is a strongly nonlinear theory of electrodynamics which allows to study in a semi-analytical way the possible creation and amplification of a primordial magnetic field during de Sitter inflation. This is so since on the one hand the lagrangian only depends on one of the electromagnetic invariants, namely $X=\frac{1}{4} F_{\mu \nu} F^{\mu \nu}$, which leads to a significant simplification of the equations. On the other hand the power-law structure of the lagrangian make the equations simpler.

Approximate solutions have been found in three regimes of approximation which describe the evolution of the ratio of the energy densities of the electric and magnetic fields during inflation. It is assumed that initially the energy density of the electric and magnetic field are of the same order. Furthermore, these initial fields are due to quantum fluctuations in the electromagnetic field during inflation. Whereas in the radiation dominated era, the energy density in the magnetic field decreases as $a^{-4}$, the electric field strength rapidly decays in the highly conducting plasma (see, e.g., [4, 25]). Solutions in closed form have been found and the resulting primordial magnetic field estimated. It has been shown that depending on the regime of approximation and the value of the Pagels-Tomboulis parameter $\delta$ primordial magnetic fields can be generated that are strong enough to seed a galactic dynamo. Thus we have provided an example of a theory of nonlinear electrodynamics where the nonlinearities act in a way as to amplify sufficiently an initial magnetic field.

The energy-momentum tensor of the electromagnetic field can be cast in the form of an imperfect fluid. This has been found explicitly for the particular model of nonlinear electrodynamics under consideration here. Moreover, this allows to find the expression for the energy density $\rho$ of the fluid. Requiring that $\rho$ should be positive provides the bound $\delta \geq \frac{1}{2}$.

In 29] the possible creation and amplification of magnetic fields was studied in an inflationary model coupled to a pseudo Goldstone boson (see also [4]). In this case the lagrangian has the form $L \sim \frac{1}{2} \partial_{\mu} \theta \partial^{\mu} \theta-X+g_{a} \theta Y$, where $\theta$ is the axion field. This provides an example of a more general lagrangian having also an explicit dependence on $Y=\frac{1}{4} F_{\mu \nu}{ }^{*} F^{\mu \nu}$. However, as it turns out the resulting primordial magnetic field is not strong enough in order to seed, for example, a galactic dynamo. In [30] the model of [29] was generalized to $\mathrm{N}$ axions. In this case it was found that at least the weaker bound of $r>10^{-57}$ can be satisfied. Here, in this work the creation of primordial magnetic fields in a particular model of nonlinear electrodynamics has been studied. It might be interesting to generalize this to lagrangians depending on both electromagnetic invariants $X$ and $Y$.

\section{Acknowledgements}

I would like to thank M. A. Vázquez-Mozo for enlightening discussions. I am grateful to the CERN theory division for hospitality where part of this work was done. This work has been supported by the "Ramón y Cajal" programme of the MEC (Spain). Partial support by Spanish Science Ministry grants FPA2005-04823 and FIS2006-05319 is gratefully acknowledged. 


\section{Appendix A}

In this section it is checked that the approximate exact solution (3.6) is a good approximation to the solution of the full differential equation (3.2). The solution (3.6) satisfies equation (3.5). Writing the full differential equation (3.2) as

$$
y \ddot{y}=\delta \dot{y}^{2}+\frac{m^{2}}{1-\delta} y^{2}+I,
$$

where for the approximate solution $y=C_{2} \cosh (z)^{\frac{1}{1-\delta}}$ with $z \equiv m\left(x+(\delta-1) C_{1}\right)$ the additional term $I$ is given by

$$
\begin{aligned}
I & \equiv \frac{C_{2}^{2 \delta+1}}{\alpha_{1}(\delta-1)} \cosh ^{\frac{2 \delta+1}{1-\delta}}(z)\left(\frac{x}{x_{1}}\right)^{-4}\left[(2 \delta-1) \frac{m^{2}}{(1-\delta)^{2}} \tanh ^{2}(z)\right. \\
& \left.-\frac{4(\delta+1)}{x_{1}}\left(\frac{x}{x_{1}}\right)^{-1} \frac{m}{1-\delta} \tanh (z)+\frac{20}{x_{1}^{2}}\left(\frac{x}{x_{1}}\right)^{-2}+\frac{m^{2}}{1-\delta}\right] .
\end{aligned}
$$

At $x_{2}$ when the comoving length scale $\lambda$ leaves the horizon $z=0$ by construction. Thus $I$ is proportional to $\left(\frac{x_{2}}{x_{1}}\right)^{-4} \ll 1$. At the end of inflation, $x=x_{1}$, using the bound on $-m x_{1}$ which in general implies $-m x_{1} \ll 1, I\left(x_{1}\right)$ is given approximately by

$$
I\left(x_{1}\right) \sim \frac{20 C_{2}^{2 \delta+1}}{(\delta-1) \alpha_{1} x_{1}^{2}} \cosh ^{-\frac{2 \delta+1}{\delta-1}}\left(z_{1}\right),
$$

where the last factor is much less than 1 since it is assumed that $\delta>1$ and, moreover, $z_{1} \sim$ $-m x_{1} e^{N(\lambda)} \gg 1$. Thus choosing $C_{2}$ appropriately, $\left|I\left(x_{1}\right)\right| \ll 1$.

Finally, it can also be checked using the bounds on $-m x_{1}$ that the square of the magnetic field strength $\vec{B}_{k}^{2}$ is well approximated by equation (3.8).

\section{Appendix B}

Expressions for $\frac{L_{X}^{\prime}}{L_{X}}$ and $\frac{L_{X}^{\prime \prime}}{L_{X}}$ for the solution (3.30).

$$
\begin{aligned}
\frac{L_{X}^{\prime}}{L_{X}}= & -\frac{2}{\eta_{1}}\left(\frac{\eta}{\eta_{1}}\right)^{-1}-3 \frac{\mu}{\eta_{1}}\left(\frac{\eta}{\eta_{1}}\right)^{2} \tanh \left[\mu\left(\frac{\eta}{\eta_{1}}\right)^{3}-c_{1}\right] \\
\frac{L_{X}^{\prime \prime}}{L_{X}}= & \frac{6}{\eta_{1}^{2}}\left(\frac{\eta}{\eta_{1}}\right)^{-2}-6 \frac{\mu}{\eta_{1}^{2}}\left(\frac{\eta}{\eta_{1}}\right) \tanh \left[\mu\left(\frac{\eta}{\eta_{1}}\right)^{3}-c_{1}\right] \\
& +9 \frac{\mu^{2}}{\eta_{1}^{2}}\left(\frac{\eta}{\eta_{1}}\right)^{4}-18 \frac{\mu^{2}}{\eta_{1}^{2}}\left(\frac{\eta}{\eta_{1}}\right)^{4} \cosh ^{-2}\left[\mu\left(\frac{\eta}{\eta_{1}}\right)^{3}-c_{1}\right],
\end{aligned}
$$

where $\mu \equiv-\left(\frac{\alpha_{2}}{18}\right)^{\frac{1}{2}} x_{1}$. 


\section{References}

[1] P.P. Kronberg, Rep. Prog. Phys. 57 (1994) 325; E. G. Zweibel and C. Heiles, Nature 385 (1997) 131; L. M. Widrow, Rev. Mod. Phys. 74 (2002) 775; M. Giovannini, Int. J. Mod. Phys. D 13 (2004) 391; R. Wielebinski and R. Beck (Eds.) "Cosmic Magnetic Fields", Lect. Notes Phys. 664 (Springer, Berlin Heidelberg, 2005); M. Giovannini, "Magnetic fields, strings and cosmology," arXiv:astro-ph/0612378.

[2] M.J. Rees, Q.J.R. Astron. Soc. 28 (1987) 197.

[3] D. Grasso and H. R. Rubinstein, Phys. Rept. 348 (2001) 163; A. D. Dolgov, "Magnetic fields in cosmology," arXiv:astro-ph/0306443.

[4] M. S. Turner and L. M. Widrow, Phys. Rev. D 37 (1988) 2743.

[5] B. Ratra, Astrophys. J. 391 (1992) L1; D. Lemoine and M. Lemoine, Phys. Rev. D 52 (1995) 1955; M. Gasperini, M. Giovannini and G. Veneziano, Phys. Rev. Lett. 75 (1995) 3796.

[6] O. Bertolami and D. F. Mota, Phys. Lett. B 455 (1999) 96; A. Ashoorioon and R. B. Mann, Phys. Rev. D 71 (2005) 103509.

[7] M. Giovannini, Phys. Rev. D 62 (2000) 123505; K. E. Kunze, Phys. Lett. B 623 (2005) 1.

[8] F. D. Mazzitelli and F. M. Spedalieri, Phys. Rev. D 52 (1995) 6694; C. G. Tsagas and A. Kandus, Phys. Rev. D 71 (2005) 123506.

[9] M. Born, Nature 132 (1933) 282; Proc. Roy. Soc. A 143 (1934) 410; M. Born and L. Infeld, Nature 132 (1933) 970 and 1004; Proc. Roy. Soc. A 144 (1934) 425.

[10] W. Heisenberg and H. Euler, Z. Physik 98 (1936) 714.

[11] J. Schwinger, Phys. Rev. 82 (1951) 664.

[12] Z. Bialynicka-Birula and I. Bialynicki-Birula, Phys. Rev. D 2 (1970) 2341.

[13] S. L. Adler, J. N. Bahcall, C. G. Callan and M. N. Rosenbluth, Phys. Rev. Lett. 25, 1061 (1970). S. L. Adler, Annals Phys. 67 (1971) 599.

[14] A. K. Harding, M. G. Baring and P. L. Gonthier, Astrophys. J. 476 (1997) 246; M. G. Baring and A. K. Harding, Astrophys. J. Lett. 507 (1998) L55; M. G. Baring, Phys. Rev. D 62 (2000) 016003; A. K. Harding and D. Lai, Rept. Prog. Phys. 69 (2006) 2631.

[15] E. S. Fradkin and A. A. Tseytlin, Phys. Lett. B 163 (1985) 123; A. A. Tseytlin, "Born-Infeld action, supersymmetry and string theory," arXiv:hep-th/9908105.

[16] G. W. Gibbons and C. A. R. Herdeiro, Class. Quant. Grav. 18 (2001) 1677; Phys. Rev. D 63 (2001) 064006.

[17] R. G. Leigh, Mod. Phys. Lett. A 4 (1989) 2767.

[18] J. Plebański, "Lectures on non-linear electrodynamics", (NORDITA, Copenhagen, 1970).

[19] G. I. Rigopoulos, E. P. S. Shellard and B. J. W. van Tent, Phys. Rev. D 73 (2006) 083521. 
[20] A. A. Starobinsky, In *De Vega, H.j. (Ed.), Sanchez, N. ( Ed.): Field Theory, Quantum Gravity and Strings*, 10\%-126; A. S. Goncharov and A. D. Linde, Sov. Phys. JETP 65 (1987) 635; H. E. Kandrup, Phys. Rev. D 39 (1989) 2245.

[21] H. Arodz, M. Slusarczyk and A. Wereszczynski, Acta Phys. Polon. B 32 (2001) 2155; M. Slusarczyk and A. Wereszczynski, Acta Phys. Polon. B 34 (2003) 2623.

[22] H. Pagels and E. Tomboulis, Nucl. Phys. B 143 (1978) 485.

[23] G. F. R. Ellis, "Cargèse Lectures in Physics" Vol.6, ed. E. Schatzman (New York: Gordon and Breach) p1; C. G. Tsagas and J. D. Barrow, Class. Quant. Grav. 14 (1997) 2539; C. G. Tsagas, Class. Quant. Grav. 22 (2005) 393; J. D. Barrow, R. Maartens and C. G. Tsagas, Phys. Rept. 449 (2007) 131.

[24] K. Subramanian and J. D. Barrow, Phys. Rev. D 58 (1998) 083502.

[25] A. Dolgov, Phys. Rev. D 48 (1993) 2499.

[26] A. C. Davis, M. Lilley and O. Tornkvist, Phys. Rev. D 60 (1999) 021301.

[27] S. Hannestad, Phys. Rev. D 70 (2004) 043506.

[28] M. C. Bento, O. Bertolami and N. J. Nunes, Phys. Lett. B 427 (1998) 261; M. C. Bento and O. Bertolami, Phys. Lett. B 365 (1996) 59.

[29] W. D. Garretson, G. B. Field and S. M. Carroll, Phys. Rev. D 46 (1992) 5346.

[30] M. M. Anber and L. Sorbo, JCAP 0610 (2006) 018. 